Revue

de Sémantique

et Pragmatique
Revue de Sémantique et Pragmatique

38 | 2015

Le futur

\title{
Quand le futur ne porte pas sur le procès qu'il actualise : futur d'énonciation et futur de découverte
}

Sophie Azzopardi et Jacques Bres

\section{OpenEdition}

\section{Journals}

Édition électronique

URL : http://journals.openedition.org/rsp/892

DOI : $10.4000 /$ rsp. 892

ISSN : 2610-4377

Éditeur

Presses universitaires d'Orléans

\section{Édition imprimée}

Date de publication : 1 septembre 2016

Pagination : 77-95

ISSN : 1285-4093

\section{Référence électronique}

Sophie Azzopardi et Jacques Bres, «Quand le futur ne porte pas sur le procès qu'il actualise : futur d'énonciation et futur de découverte », Revue de Sémantique et Pragmatique [En ligne], 38 | 2015, mis en ligne le 01 février 2016, consulté le 11 avril 2020. URL : http://journals.openedition.org/rsp/892 ; DOI : https://doi.org/10.4000/rsp.892 


\title{
Quand le futur ne porte pas sur le procès qu'il actualise : futur d'énonciation et futur de découverte
}

\author{
Sophie Azzopardi et Jacques Bres
}

1 Le futur est un temps verbal qui, du fait de sa valeur en langue, semble prédisposé à développer des emplois différents de l'emploi temporel prototypique d'ultérieur du PRÉSENT ${ }^{1}$, qualifiés de « modaux » par la tradition grammaticale ${ }^{2}$. Notre travail portera sur deux emplois qui, à notre connaissance, n'ont pas fait l'objet de travaux spécifiques. Distincts de l'emploi temporel prototypique, ils ne relèvent pas pour autant de la catégorie de la modalité définie comme " expression de l'attitude du locuteurénonciateur par rapport au contenu propositionnel de l'énoncé ». En raison de leur sémantisme, qui sera explicité infra, nous les qualifierons d'emplois énonciativotemporels et les nommons provisoirement emploi d'énonciation et emploi de découverte. Le premier concerne le futur simple (1) et le futur antérieur (2); le second, le futur simple (3) et le futur périphrastique (4) :

1. Mais n'est-il pas plus simple que j'aille à Paris ? Ma mère pourra trouver un prétexte pour m'y envoyer : ce sera un oncle qui me demande, une tante en train de mourir, une dame qui me voudra du bien. (Balzac, La Vieille fille, 1836)

2. - on voit bien, dit l' abbé Pirard, que vous habitez Paris. [...] On ne voudra pas laisser partir Julien Sorel, on saura se couvrir des prétextes les plus habiles, on me répondra qu'il est malade, la poste aura perdu les lettres, etc., etc. (Stendhal, Le Rouge et le noir, 1830)

3. (à un client qui lui demande un type de chaussures, une vendeuse répond) : elles seront sur ce présentoir là-bas (apud Saussure et Morency 2012)

4. (une cliente d'une épicerie bio s'adresse à une vendeuse en train de ranger un rayon) :

- bonsoir / je cherche les oeufs s'il vous plaît

- alors / ça va être dans l'allée du frais / à côté du rayon surgelé / là-bas à gauche (juillet 2014)

2 Ces deux emplois ont été réunis ici parce qu'ils partagent au moins trois caractéristiques. La première concerne le type de discours duquel ils relèvent puisqu'on les rencontre dans des conversations authentiques (discours conversationnel effectif) ou dans des dialogues littéraires (discours conversationnel représenté). La deuxième a trait à la localisation du procès dénoté au futur : il est situé dans le PRÉSENT, et non 
dans le futur. Pour autant, dans les deux cas, il ne s'agit pas d'emplois modaux en ce sens qu'à la différence, par exemple, de l'emploi conjectural, ils n'expriment pas l'attitude du locuteur-énonciateur au sujet du contenu propositionnel de l'énoncé. La troisième enfin, est inhérente au fonctionnement du futur qui dans les deux cas, bien que de manière différente, ne porte pas sur le procès qu'il actualise. Ces emplois sont à première vue peu fréquents, ce qui explique peut-être qu'ils aient pu passer presque incognito des linguistes - nous avons seulement trouvé une rapide mention du premier dans Martin (1981) et dans Vuillaume (1998) ; l'exemple (3), quant à lui, est proposé par Saussure et Morency (2012) qui en font une analyse différente de la nôtre. Dans le corpus de 149 énoncés au futur simple et au futur antérieur en français issus de différents genres discursifs (conversation, littérature, presse, internet) constitué dans le cadre des travaux présentés dans Azzopardi (2011), seuls 4 énoncés contenaient un futur en emploi d'énonciation et aucun emploi de découverte n'avait été relevé. L'analyse exposée ici se situe au commencement d'un travail de recherche portant spécifiquement sur ces deux emplois et de ce fait, le corpus constitué en vue de cette étude est composé d'occurrences relevées " au vol » ou au fil de nos lectures. Il se compose :

- pour l'emploi d'énonciation, de 13 occurrences textuelles réalisant un total de 22 verbes au futur (9 au futur simple, 13 au futur antérieur) ;

- pour l'emploi de découverte de 4 occurrences au futur simple et 1 au futur périphrastique.

Ce corpus ne permet donc pas pour l'heure de mener une analyse quantitative et ne prétend aucunement être représentatif de ce point de vue-là, mais demeure suffisant pour mettre en évidence certains principes de fonctionnement du mécanisme de production de sens dans ce type d'énoncés. Notre hypothèse est que, comme c'est le cas pour tous les énoncés dans lesquels un futur est employé, c'est l'interaction, dans le temps d'actualisation, de la valeur en langue du futur avec des éléments co(n)textuels différents suivant les énoncés qui permet à ce temps d'entrer dans la production d'effets de sens différents en discours : ultérieur, hypothétique, narratif, conjectural, etc., et, pour ce qui nous intéresse ici : d'énonciation et de découverte. Dans un premier temps, nous exposerons les cadres théoriques dans lesquels nous ancrons notre analyse et décrirons la valeur en langue du futur (section1.). Nous étudierons ensuite le fonctionnement de l'emploi d'énonciation (section 2.), puis celui de l'emploi de découverte (section 3.).

\section{Cadres théoriques et valeur en langue du futur}

\subsection{Actualisation, production de sens, dialogisme et temps verbaux}

4 Notre analyse s'inscrit dans les cadres d'une linguistique de l'actualisation / de la production de sens et fait appel à la notion de dialogisme envisagée d'un point de vue énonciatif. Nous considérons le sens comme le résultat d'un processus d'actualisation ${ }^{3}$ qui met en relation deux plans : d'une part, celui de la langue, où se détermine la valeur de base des morphèmes, outils de production de sens ; et d'autre part, celui du discours où se réalise la production de tel ou tel effet de sens. L'actualisation est le processus qui permet de passer de la langue au discours, par interaction de la valeur en langue des morphèmes en présence entre eux et avec le co(n)texte ${ }^{4}$. On pose donc que les divers effets de sens (ultérieur, narratif, hypothétique, conjectural, de mitigation, de bilan) produits par des énoncés au futur (simple, antérieur ou périphrastique) en discours sont le 
résultat de l'interaction d'une même valeur en langue de ce temps avec des éléments co(n)textuels différents. Dans cette perspective, le futur n'est pas considéré comme le seul responsable de la production de ces effets de sens mais comme un ingrédient qui y participe au même titre que les autres éléments du co(n)texte. Notre analyse s'attachera à mettre en évidence ces éléments et à montrer le rôle que chacun joue dans la production des effets de sens d'énonciation et de découverte. Ce travail sera conduit dans le cadre d'une linguistique de l'énonciation qui fait appel à la notion de dialogisme ${ }^{5}$, issue des travaux du cercle de Bakhtine (1952/1979/1984) et telle qu'elle a été définie par Bres \& Nowakowska (2006) : un énoncé est dialogique dès lors que l'énonciation principale [e] entre en interaction avec une énonciation secondaire [e] avec laquelle elle entretient une relation hiérarchique [e[e]]. L'énoncé se fait alors l'écho non seulement de la voix du locuteur-énonciateur principal E1, mais aussi de celle d'un énonciateur secondaire e1 qui peut être ou non coréférent à E1. Au temps de l'énonciation principale to correspond ainsi un temps de l'énonciation secondaire tn qu'il convient de prendre en compte dans le cadre d'une analyse des temps verbaux, qui, nous allons le voir pour le futur, sont concernés par la problématique dialogique (Bres 2009).

\subsection{Va leur en langue du futur (simple et antérieur) et de la forme périphrastique}

En appui sur sa construction morphologique, on pose que le futur donne deux types d'instructions : temporelles et aspectuelles ${ }^{6}$. Du point de vue temporel, on établit que le futur (simple ou antérieur) est un ultérieur du PRÉSENT. Soit le verbe voyager : [voyage - $\mathrm{r}$ - ai]. Dans l'énoncé (Plus tard) je voyagerai, le futur place le procès voyager dans l'ultériorité (instruction [+ ultériorité] issue du morphème -r-) à partir du PRÉSENT (instruction [+ PRÉSENT] issue de la terminaison -ai de l'auxiliaire avoir au présent) de l'énonciation principale [e]. L'événement est donc placé dans le FUTUR. Si futur simple et futur antérieur partagent ces instructions temporelles, ils se différencient du point de vue aspectuel. Le procès est vu soit en tension, soit en extension (Cf. Guillaume 1929/1970). Le futur simple montre le procès dans son accomplissement (instruction [+ tension]). Le futur antérieur, quant à lui, du fait du participe passé, montre le procès accompli (instruction [+ extension]). La forme périphrastique est composée de l'auxiliaire aller au présent et de l'infinitif. Par grammaticalisation du verbe de déplacement aller en auxiliaire aspectuo-temporel prospectif (Hopper et Traugott 1993/2003, Bybee et al. 1994), la périphrase, lorsque aller est au présent, situe dans le PRÉSENT de l'énonciation principale (instruction [+ PRÉSENT]) la phase préparatoire du procès (instruction [+ prospectif]). Par inférence, le procès lui-même est de ce fait situé dans l'ultériorité du PRÉSENT, à savoir le FUTUR : c'est ce qui permet d'expliquer le fait que la périphrase se trouve en concurrence directe avec le futur simple dans son emploi prototypique.

6 Soit le tableau récapitulatif suivant: 


\begin{tabular}{|c|c|c|}
\hline & Instructions temporelles & Instructions aspectuelles \\
\hline $\begin{array}{c}\text { Futur simple } \\
\text { je voyagerai }\end{array}$ & \multirow{2}{*}{ [+ ultériorité] [+ PRÉSENT] } & [+ tension] \\
\cline { 1 - 3 } $\begin{array}{c}\text { Futur antérieur } \\
\text { j'aurai voyagé }\end{array}$ & & [+ extension] \\
\hline $\begin{array}{c}\text { Futur périphrastique } \\
\text { je vais voyager }\end{array}$ & [+ PRÉSENT] & [+ prospectif] \\
\hline
\end{tabular}

7 C'est sur ces bases que nous allons analyser l'emploi d'énonciation (qui a fait l'objet d'une première analyse dans Azzopardi 2011), puis l'emploi de découverte.

\section{Emploi d'énonciation}

\subsection{Définition et description}

Rappelons ici les exemples (1 et 2) mentionnés en introduction pour illustrer l'emploi d'énonciation du futur :

1. Mais n'est-il pas plus simple que j'aille à Paris ? Ma mère pourra trouver un prétexte pour m'y envoyer : ce sera un oncle qui me demande, une tante en train de mourir, une dame qui me voudra du bien. (Balzac, La Vieille fille, 1836)

2. - on voit bien, dit l'abbé Pirard, que vous habitez Paris. [...] On ne voudra pas laisser partir Julien Sorel, on saura se couvrir des prétextes les plus habiles, on me répondra qu'il est malade, la poste aura perdu les lettres, etc., etc. (Stendhal, Le Rouge et le noir, 1830)

Décrivons cet emploi dans une perspective contrastive avec l'emploi deconjecture (5 et 6) avec lequel il semble de prime abord partager certains traits :

(5)On entendit frapper à la porte de la ruelle. Gasselin alla ouvrir. «-Ce sera sans doute M. le curé, il vient presque toujours le premier ", dit Mariotte. (Balzac H. de, Béatrix, 1839)

(6)(conversation. Jules attend Maria dans un café, elle arrive avec quelque retard, 2005) : - ah te voilà... je me disais « elle t'aura posé un lapin »

Pour ce qui est de la seule localisation temporelle des événements, le futur simple en (5) est remplaçable par le présent (5a) ; et le futur antérieur en (6), par le passé composé (6a). Les procès qu'ils actualisent sont inscrits non dans le FUTUR, mais dans le PRÉSENT pour le premier, et dans le PASSÉ pour le second.

(5a)-C'est sans doute M. le curé, il vient presque toujours le premier

(6a)ah te voilà...je me disais " elle t'a posé un lapin »

Dans l'emploi énonciatif, ce remplacement est possible mais uniquement via la médiation d'une énonciation dont le procès sera actualisé au futur simple (ou au futur périphrastique selon le co(n)texte) : le futur de (1) peut être remplacé par dira que + présent (1a), et le futur antérieur de (2) par dira que + passé composé (2a), à savoir par du discours indirect :

(1a) Mais n'est-il pas plus simple que j'aille à Paris ? Ma mère pourra trouver un prétexte pour m'y envoyer : elle dira que c'est un oncle qui me demande, une dame qui me veut du bien?

(2a) on ne voudra pas laisser partir Julien Sorel, on saura se couvrir des prétextes les plus habiles, on me répondra qu'il est malade, (on me dira) que la poste a perdu les lettres, etc., etc. 
Les occurrences (1) et (2) apparaissent comme des tours elliptiques du discours citant, qui peuvent être rapprochées d'occurrences sans ellipse comme :

(7)(Une vieille dame, Mme d'Ambérieux, refuse de se rendre à un repas de famille, et prétexte un malaise. Sa fille :) Eh bien, c'est ça, on dira que tu es souffrante. (Aragon, Les Voyageurs de l'impériale, 1942)

(8)(Une vieille femme vient de mourir. Son fils propose d'envoyer rapidement une dépêche à sa sœur pour lui faire savoir le décès de leur mère, mais sa femme l'en dissuade :) « Non, envoie-la seulement de dix à onze, afin que nous ayons le temps de nous retourner avant son arrivée. Nous dirons que tu as perdu la tête.» (Maupassant, En famille, 1881)

Poursuivons l'étude contrastive des deux tours conjectural et énonciatif :

- sémantiquement / pragmatiquement : il s'agit, dans la conjecture, de proposer une explication à un fait, explication que le locuteur prend en charge ${ }^{8}$ et qui, selon lui, sera vérifiée dans le futur ; il s'agit, dans l'énonciatif, de trouver un prétexte à un fait en maquillant sa cause ;

- énonciativement : le tour conjectural apparait dans une interaction verbale directe (6) ou rapportée (5) (avec un tiers ou avec soi-même) : le locuteur adresse l'énoncé au futur à son interlocuteur directement : en (5), Mariotte s'adresse à ses commensaux; ou, plus rarement, via la médiation d'une interaction antérieure : en (6), Jules rapporte à Maria l'énoncé qu'il s'est adressé à lui-même antérieurement.

14 Le tour énonciatif apparaît également dans une interaction verbale directe (infra 10) ou rapportée $(1,2)$; mais le locuteur adresse l'énoncé au futur à son interlocuteur toujours via la médiation d'une interaction verbale implicite et cette interaction est toujours ultérieure : en (2) p. ex. l'énoncé « la poste aura perdu les lettres » est ce que l'abbé Pirard dit au marquis de la Mole, énoncé qui rapporte par avance une interaction ultérieure (donc imaginée) dans laquelle un locuteur indéfini (on) produira l'énoncé « la poste a perdu les lettres » comme prétexte fourni à l'abbé Pirard pour expliquer la nonvenue de Julien. On a donc toujours affaire à une double interaction : citante (interaction 1), et citée (interaction 2), ultérieure, avec différentes relations possibles entre les interactants de 1 et ceux de 2 , ce qui n'est pas le cas des énoncés conjecturaux. Mentionnons les principales relations possibles en nous appuyant sur (1) et (2) ainsi que sur de nouvelles occurrences ( 9 et 10) :

- en (1) : locuteur 2 est un tiers (ma mère) et interloc.2 n'est pas précisé ;

- en (2) : loc.2 est un tiers indéfini (on), et interloc.2 coréfère avec loc.1;

- en (9) : loc.2 coréfère avec loc. 1 ; interloc. 2 est un tiers (mon mari) :

(9)(Mme de Rênal imagine un stratagème de lettre anonyme pour répondre à l'accusation d'adultère qui pèse sur elle) J'irai dans le village et reviendrai avec un visage troublé, je le serai en effet beaucoup. Grand dieu! Qu'est-ce que je hasarde, et tout cela parce que tu as cru deviner une lettre anonyme. Enfin, avec un visage renversé, je donnerai à mon mari cette lettre qu'un inconnu m'aura remise. (Stendhal, Le Rouge et le noir, 1830) ( $\rightarrow$ je dirai à mon mari qu'un inconnu me l'a remise)

- En (10), loc.2 coréfère avec interloc. 1 ; interloc. 2 coréfère avec loc.1 :

(10)Elle. N'use pas ta salive, je sais ce que tu vas me dire. (très simple). Je me suis fait peloter. Lui. Oui, tu t'es fait peloter. Elle. (assise près du lit et commençant à se dévêtir) Là. Oh ! je connais l'ordre et la marche. Dans un instant je me serai conduite comme une fille, dans deux minutes tu m'appelleras sale bête ; dans cinq tu casseras quelque chose. (Courteline G., La peur des coups, 1894 ; apud Vuillaume 1998)( $\rightarrow$ tu me diras que je me suis conduite)

15 Du fait que cet emploi implique plusieurs interactions, il ne nous semble pas possible de l'analyser en termes de polyphonie (Cf. Kronning 1993 et 2003, i.a.) ou de polychronie (Cf. Abouda 2004) puisque le fonctionnement énonciatif de ce type d'énoncés va au-delà de la distorsion temporelle entre le locuteur et l'énonciateur. De la même façon, si à 
première vue, le fait que dans l'emploi d'énonciation le futur ne porte pas sur le procès qu'il actualise aurait pu laisser penser qu'une analyse en termes d'opposition entre temporalité de re et temporalité de dicto (Cf. Martin 1985, Vetters 2001) permettait d'expliquer le fonctionnement de cet effet de sens, il n'en est rien. L'opposition entre ces deux types de temporalité est par exemple utilisée par Vetters (2001 : 181) pour expliquer l'emploi narratif du futur :

«De re, les faits relatés sont situés dans le passé, mais de dicto, ils sont pris en charge comme s'ils étaient des faits futurs. » (Vetters 2001, 181)

Dans un énoncé comme « [Christian] Bourgois ira à Science-Po et en sortira second de sa promo (devant Jacques Chirac); puis, il entrera à l'ENA... qu'il quitte au bout d'un an. " (Nova Planet, 20/12/2007), on peut dire qu'il y a un décalage entre temporalité de re (les événements sont antérieurs au moment de l'énonciation principale) et temporalité de dicto (les événements sont présentés comme ultérieurs). Or, on n'assiste pas à un décalage de ce type dans l'emploi d'énonciation. Dans (1) par exemple, le procès être de " ce sera un oncle qui me demande ", n'est pas situé de re, puisque le locuteurénonciateur n'en affirme pas l'existence, et il est situé de dicto comme simultané à une interaction distincte de l'interaction principale et ultérieure à celle-ci. Indirectement, on peut inférer de cela que le procès être est ultérieur à l'énonciation principale, à la différence près que son existence ne peut être posée qu'au travers de la médiation d'une seconde interaction. Nous complèterons cette description de l'emploi d'énonciation du futur en apportant des précisions sur (i) son sémantisme, (ii) ses réalisations et (iii) sa datation.

(i)De par l'analyse proposée jusqu'à présent, on pourrait catégoriser l'énoncé au futur comme réalisant un acte de parole mensonger, mensonge imaginé par loc.1 et prêté à loc.2. Cette analyse serait partielle : si effectivement ledit acte relève du mensonge dans 9/13 occurrences du corpus (ce qui est le cas en (1), (2), (9)), s'il se voit qualifié par le cotexte à deux reprises de "prétexte ", il n'en va pas toujours ainsi. Dans 4 occurrences, l'acte de parole imaginé par loc.1 est présenté non pas comme un prétexte trouvé par loc. 2 pour maquiller la cause d'un fait, mais comme correspondant à ce que pense effectivement loc.2, dont loc.1 laisse entendre le caractère fallacieux : ainsi en (10), Elle prête à Lui, à travers l'énoncé « Dans un instant je me serai conduite comme une fille ", non un propos mensonger mais un propos qui correspond à ce que Lui pense et qui, selon Elle, ne correspond pas à la réalité. Ce qu'ont de commun toutes les occurrences du corpus, c'est que loc.1 se désolidarise de la parole qu'il impute à loc.2 en la montrant mensongère, erronée, voire totalement absurde comme en (11) :

(11)LE PÈRE - Et quand tu nous reviendras le ventre gonflé, ayant déshonoré le nom de ton père, tué ta mère de douleur, et forcé tes frères à s'engager dans l'armée pour fuir la honte au village - ce sera le Saint-Esprit, peut-être, qui aura fait le coup ! Je vais le dire au curé que, non contente de courir, tu blasphèmes. On te jettera, au pain et à l'eau, à moisir dans un cul de couvent! (Anouilh J., L'Alouette, 1953) ( $\rightarrow$ tu diras que c'est le Saint-Esprit, peut-être, qui a fait le coup !)

(ii)Les occurrences présentées jusqu'à présent relèvent toutes de l'écrit littéraire (roman, théâtre). Serait-ce que l'oral conversationnel ne connaîtrait pas le futur énonciatif ? Nous le pensions... jusqu'au jour où nous avons pu relever « au vol » cette occurrence :

(12)(conversation d'une vieille dame (Juliana) avec son fils (Pierre) : elle lui parle d'un cousin, Gaston, qui perdait la tête pour les jolies femmes (2014)) [...] c'est comme la fois où Lucette lui a demandé de la raccompagner à Alès / il a dit « oui bien sûr » mais on devait ce jour-là aller à la mer l'après-midi / sa fille [Odette] était folle « oui après il sera fatigué il aura mal à la tête il faudra qu'il fasse sa sieste » et 
puis non on est allés à la mer quand même ${ }^{9}\left(\rightarrow\right.$ Il dira $q u^{\prime}$ il est fatigué, $q u u^{\prime}$ il $\boldsymbol{a}$ mal à la tête, $q u$ 'il faut qu'il fasse sa sieste)

(iii)Est-il possible de dater cet emploi du futur ? Est-il apparu seulement au XIXe comme on pourrait l'inférer des occurrences antérieures? Il semble être entré dans la langue au moins depuis le XVIIe, comme l'atteste (13):

(13)

- AGRIPPINE. Arrêtez, Néron : j'ai deux mots à vous dire. / Britannicus est mort, je reconnois les coups

; /Je connois l'assassin.

- NÉRON. Et qui, Madame?

- AGRIPPINE. Vous.

- NÉRON. Moi ! Voilà les soupçons dont vous êtes capable./ Il n'est point de malheurs dont je ne sois coupable ; / Et si l'on veut, Madame, écouter vos discours,/ Ma main de Claude même aura tranché les jours./ Son fils vous étoit cher : sa mort peut vous confondre ; / Mais des coups du destin je ne puis pas répondre. (Racine, Britannicus, 1669) ( $\rightarrow$ Vous direz que ma main de Claude même a tranché les jours)

Nous venons de décrire l'emploi d'énonciation du futur. Il nous reste maintenant à proposer une hypothèse explicative de son fonctionnement.

\subsection{Explication}

Nous avons vu que la valeur en langue du futur [+ ultériorité] [+ PRÉSENT] actualisait prototypiquement le procès de l'énoncé. Dans l'emploi d'énonciation, le co(n)texte empêche que ces instructions se portent sur cet élément pour des raisons de cohérence temporelle et énonciative. Prenons seulement (10), "Dans un instant je me serai conduite comme une fille » : le futur antérieur ne peut, malgré le circonstant dans un instant, poser comme ultérieur par rapport à to le procès se conduire comme une fille, qui cotextuellement correspond à l'évaluation du procès antérieur se faire peloter. D'autre part, énonciativement, Elle ne peut être l'énonciateur de « je me serai conduite comme une fille » : elle ne prend pas en charge ce procès. Ainsi contraintes, les instructions temporelles du futur se reportent sur une énonciation (implicite) ultérieure, en interaction concordante avec les éléments co(n)textuels temporellement et énonciativement : la mise en place d'un acte de parole ultérieur s'accorde avec le circonstant dans un instant. Il se voit confirmé par l'explicitation d'un acte de parole symétrique dans l'énoncé qui suit : «dans deux minutes tu m'appelleras sale bête ». Et énonciativement, il peut parfaitement être pris en charge par l'énonciateur Lui. Ce fonctionnement du futur que nous avons nommé énonciatif parce qu'il consiste à faire porter les instructions de ce temps non sur le procès de l'énoncé mais sur son énonciation est explicable en termes de coercion (Pustejovsky 1995; de Swart 1998), d' usage interprétatif (Sperber et Wilson 1995), ou de dialogisme (Azzopardi et Bres 2011). C'est cette dernière approche que nous développons. Nous avons défini le futur comme un ultérieur du PRÉSENT : l'ultériorité du procès, du fait qu'elle se calcule à partir de to, a pour origine le seul locuteur-énonciateur E1 : le futur n'est pas un temps dialogique en langue, à savoir qu'il ne présuppose pas, à côté du locuteur-énonciateur E1, un énonciateur secondaire e1 (cas du conditionnel). Mais il peut avoir un fonctionnement dialogique en discours, lorsque le co(n)texte l'y oblige, comme dans les emplois de mitigation, de conjecture, de bilan (Azzopardi 2011, Azzopardi et Bres 2011), et donc dans l'emploi d'énonciation. Reprenons encore une fois (10) en remplaçant le futur antérieur par le passé composé :

(10a)Elle. (assise près du lit et commençant à se dévêtir) Là. Oh ! je connais l'ordre et la marche. Dans un instant je me suis conduite comme une fille, dans deux minutes tu m'appelleras sale bête; dans cinq tu casseras quelque chose. 
L'interprétation de l'énoncé devient problématique : le passé composé me suis conduite se combine difficilement avec le circonstant dans un instant, l'énonciation implicite est difficilement accessible. C'est bien le futur, qui, en interaction avec le co(n)texte, permet de comprendre que Elle place là implicitement un acte d'énonciation ultérieur attribué à $L u i^{10}$. Les paraphrases par lesquelles nous avons décrit l'emploi d'énonciation conduisent à le mettre en relation avec la problématique du discours indirectlibre. M. Vuillaume termine l'article qu'il a consacré à cette question de lasorte :

«Il s'ensuit que seules les formes verbales en -ait - imparfait, plus-que-parfait et conditionnel -, le futur antérieur et (peut-être) le futur simple sont aptes à marquer, dans le D.I.L., le décalage entre le moment de la production des propos mentionnés et celui où on les mentionne. " (Vuillaume 1998, 200)

21 Nous confirmons cette analyse ${ }^{11}$ : le futur antérieur mais aussi le futur simple - le doute de M. Vuillaume à l'endroit de ce temps peut être levé : il se voit réalisé par $9 / 22$ occurrences (notamment (1), (12)) de notre corpus -, peuvent être utilisés en discours indirect libre. Ils permettent d'actualiser indirectement des propos comme relevant d'un énonciateur e1 autre que l'énonciateur principal E1 sans mention du discours citant. Nous ajouterons toutefois une précision : les occurrences de futur énonciatif ne le font pas tout à fait comme les autres. Prenons une occurrence prototypique de D.I.L. au conditionnel :

(14)Il s'allongea, les yeux au plafond, ébloui par la forte clarté neutre et morte qui remplissait la chambre. Il remonterait là-haut à la nuit tombante. Peut-être irait-il prendre un affût. (Carrière, L'épervier de Maheux, 1972)

L'ultériorité porte non sur l'énonciation mais sur le procès : c'est dans le moment où l'actant est allongé qu'il se dit (énonciation) qu'il fera ceci et cela plus tard (procès). Et il en va de même si nous transposons le fragment textuel en narration présente :

(14a)Il s'allonge, les yeux au plafond (...). Il remontera là-haut à la nuit tombante. Peut-être ira-t-il prendre un affût.

Ce qui donne, en explicitation de l'acte d'énonciation :

(14b)Il s'allonge, les yeux au plafond (...). Il se dit qu'il remontera là-haut à la nuit tombante, qu'il ira peut-être prendre un affût.

L'énonciation est simultanée au cotexte antérieur, comme le signale le présent ; c'est le procès qui est placé en ultériorité, comme le signale le futur. Dans l'emploi d'énonciation du futur, c'est l'énonciation rapportée qui est placée en ultériorité, comme le fait apparaître le test de remplacement :

(10)Elle. (assise près du lit et commençant à se dévêtir) Là. Oh ! je connais l'ordre et la marche. Dans un instant je me serai conduite comme une fille, dans deux minutes tu m'appelleras sale bête ; dans cinq tu casseras quelque chose. (Courteline G., La peur des coups, apud Vuillaume 1998) ( $\rightarrow$ tu diras que je me suis conduite comme une fille)

Ce qui est actualisé au futur, c'est non le procès mais l'énonciation de ce procès qui, lui, est actualisé au passé composé. Nous ne disposons d'aucune occurrence au futur périphrastique : est-ce à dire que cette forme serait ici mal venue, voire impossible ? Il semble que dans l'occurrence (12) on puisse remplacer les futurs simples par va + inf; et en (10),

le futur antérieur par va+infinitif composé:

(12a)[...] sa fille [Odette] était folle « oui après il va être fatigué il va avoir mal à la

tête il va falloir qu'il fasse sa sieste » et puis non on est allés à la mer quand même 
(10a)[...] Oh ! je connais l'ordre et la marche. Dans un instant je me vais m'être conduite comme une fille. (15 et 16) issues de notre corpus :

(3)(à un client qui lui demande un type de chaussures, une vendeuse répond) : elles seront sur ce présentoir là-bas (apud Saussure et Morency 2012)

(4)(une cliente d'une épicerie bio s'adresse à une vendeuse en train de ranger un rayon):

. bonsoir / je cherche les oeufs s'il vous plaît

- alors / ça va être dans l'allée du frais / à côté du rayon surgelé / là-bas à gauche (juillet 2014)

(15)(Boutique de vêtements. Une cliente cherche une chemise dans une colonne)

-quelle taille vous cherchez?

- M

- alors oui / on aura mais ce sera dans cette colonne (corpus Bres, déc. 2013)

(16)(Une vieille dame au moment de sortir promener)

- Ma canne... mince... je l'avais ce matin là derrière... ah bé je l'ai oubliée dans le garage / chaque fois je fais le coup / Pierre tu vas me la chercher ? elle sera contre les outils en rentrant là à droite (corpus Bres, mars 2014)

Saussure \& Morency (2012) et Saussure (2012) analysent l'occurence (3) comme relevant de la modalité épistémique, au même titre que (5) que nous rappelons :

(5)On entendit frapper à la porte de la ruelle. Gasselin alla ouvrir. « - Ce sera sans doute M. le curé, il vient presque toujours le premier ", dit Mariotte. (Balzac H. de, Béatrix, 1839)

Pour ce faire, ils distinguent deux types d'emploi épistémique du futur :

- celui qui correspond à l'expression d'une " évidentialité inférentielle » comme en (5), emploi que, suivant la tradition, nous avons nommé conjectural, et qui exprime une hypothèse visant à expliquer la cause d'un fait ;

- celui qui correspond à l'expression d'une probabilité ou d'une possibilité devant être vérifiée, comme en (3) :

« [...] le futur épistémique n'exprime pas toujours l'évidentialité inférentielle de l'explication causale d'un fait perçu (le coup de sonnette et sa cause par ex.). Demandant à une vendeuse (dans le centre de la France) si son magasin vendait des chaussures d'une certaine marque, je me suis entendu répondre [...] : Elles seront sur ce présentoir là-bas. [Cet énoncé] ne manifeste aucun raisonnement à propos des causes d'une quelconque perception (il n'y en a pas dans les circonstances, 
hormis ma parole, qui ne saurait être causée par la présence de chaussures ici ou là). » (Saussure 2012, 135)

«[...] l'énoncé exprime tout simplement quelque chose qui peut ressembler à « Je crois me souvenir qu'elles sont sur ce présentoir », assorti de l'effet de vérification future qui viendra prouver la validité du souvenir. » (Saussure 2012 : 136, note 3)

Nous ne partageons pas cette analyse : selon nous les énoncés des occurrences (3), (4), (15) et (16) n'ont pas une valeur modale épistémique en ce sens d'une part qu'ils n'expriment pas une hypothèse visant à caractériser une situation ou à en expliquer la cause, et d'autre part qu'ils ne présentent pas l'événement dénoté par le verbe comme simplement possible ou probable. Ils produisent un effet de sens spécifique - de découverte -, différent de l'effet de sens de conjecture, duquel nous allons le distinguer contrastivement. Le premier point que l'on peut mettre en évidence concerne les formes de futur qui peuvent intervenir dans les énoncés conjecturaux et dans les énoncés de découverte. Les énoncés conjecturaux peuvent être produits au futur simple avec être et avoir et de façon beaucoup plus fréquente avec tous les verbes au futur antérieur. Sur un corpus de 69 énoncés conjecturaux au futur en français provenant de discours écrits ou oraux et appartenant à divers genres comme la littérature ou la conversation (cf. Azzopardi 2011), 50 sont au futur antérieur alors que 19 seulement sont au futur simple. Par ailleurs, aucun n'est réalisé à la forme périphrastique en aller + infinitif. Les énoncés de découverte, quant à eux, ne sont produits qu'avec les verbes d'état être et avoir, au futur simple ou à la forme périphrastique et nullement au futur antérieur. Nous proposerons une explication à ce double phénomène infra (point 3.2.).

Du point de vue de la localisation temporelle des événements, le futur de découverte peut être remplacé par le présent, comme c'est le cas dans l'emploi de conjecture:

(3a)elles sont sur ce présentoir là-bas.

(4a)alors / c'est dans l'allée du frais / à côté du rayon surgelé / là-bas à gauche.

(15a)- alors oui / on a mais c'est dans cette colonne (corpus Bres, nov-déc. 2013)

(16a)Ma canne... mince... je l'avais ce matin là derrière... ah bé je l'ai oubliée dans le

garage / chaque fois je fais le coup / Pierre tu vas me la chercher ? elle est contre

les outils en rentrant là à droite

Cependant, contrairement à l'emploi conjectural, le locuteur n'y formule pas une hypothèse sur le lieu où peut se trouver l'objet recherché ou visant à expliquer pourquoi il se trouve là. Dans ces quatre occurrences, le locuteur sait parfaitement où se trouve l'objet en question et le fait qu'il s'y trouve n'a rien de surprenant ni pour lui ni pour son interlocuteur : nous n'y voyons donc aucune valeur modale épistémique. On peut remarquer en revanche que les énoncés de découverte se caractérisent par le trait suivant, absent dans l'expression de la conjecture : ils font toujours partie d'une interaction verbale, ce qui n'est pas forcément le cas des énoncés conjecturaux, interaction dans laquelle le locuteur indique à l'interlocuteur l'endroit où il entrera en contact (visuel) avec l'objet recherché au terme d'un déplacement (les chaussures en 3, les œufs en 4 , la chemise en taille $\mathrm{M}$ en 15 et la canne en 16). Cette caractéristique nous invite à faire un parallèle entre les énoncés de découverte et l'exemple (17) issu d'un corpus d'indications d'itinéraires :

(17)

.E3 - pardon monsieur / l'église Saint Roch s'il vous plaît

- 2.I - euh:: alors Saint Roch euh:: oui attende:z alors venez : (commence à remonter la rue des Etuves, suivi par E2 et E3) (...) vous allez voir / vous allez prendre un peu à droite / (hm E2) i va y avoir la Chambre de Commerce avec <écrit> CCI / tout de suite à gauche / (oui E2) (corpus Barbéris) 

production de l'effet de sens de découverte : une interaction verbale dans laquelle E1 indique l'endroit où l'interlocuteur entrera ultérieurement en contact (visuel) avec l'objet recherché et des éléments qui signalent que l'objet recherché se situe déjà à cet endroit-là. Dans son emploi temporel prototypique, les instructions temporelles du futur [+ ultériorité] et [+ PRÉSENT] portent sur le procès au futur et le situent dans l'ultériorité de t0. La forme périphrastique, quant à elle, place dans le PRÉSENT la phase préparatoire d'un procès dont le déroulement est situé dans l'ultériorité de to. Dans le cas des emplois de découverte, alors même qu'est mis au futur le procès qui localise l'objet, le co(n)texte signale implicitement que l'objet se trouve à cet endroit au moment to de l'énonciation principale et non dans son ultériorité : les chaussures en (3), les oeufs en (4), la chemise en (15) comme la canne en (16) sont déjà sur le présentoir, dans l'allée du frais, dans la colonne voisine ou dans le garage au moment de l'énonciation, et le locuteur en a déjà connaissance. Ce que le futur, de par sa valeur en langue, situe dans l'ultériorité du moment de l'énonciation principale, c'est donc non la localisation de l'objet recherché mais le moment où l'interlocuteur entrera en contact visuel avec lui, ce que l'on peut expliciter de la sorte :

(3)elles seront sur ce présentoir là-bas. ( $\rightarrow$ vous les trouverez / allez les trouver sur le présentoir là-bas)

(4)- alors / ça va être dans l'allée du frais / à côté du rayon surgelé / là-bas à gauche. ( $\rightarrow$ vous allez les trouver / les trouverez dans l'allée du frais à côté du rayon surgelé là-bas à gauche.)

(15)- alors oui / on aura mais ce sera dans cette colonne ( $\rightarrow$ vous les trouverez / allez les trouver dans cette colonne)

(16)- Ma canne... mince... je l'avais ce matin là derrière... ah bé je l'ai oubliée dans le garage / chaque fois je fais le coup / Pierre tu vas me la chercher ? elle sera contre les outils en rentrant là à droite $(\rightarrow$ tu la trouveras / vas la trouver contre les outils en rentrant là à droite)

Cette explicitation permet de remarquer que dans ce type d'emploi, le futur simple et la forme périphrastique sont deux formes quasi interchangeables, comme c'est le cas dans l'emploi temporel prototypique. C'est un élément qui vient conforter notre analyse selon laquelle l'emploi de découverte est bien distinct de l'emploi conjectural. Dans l'emploi de découverte, le futur n'a pas une valeur modale mais bien une valeur temporelle. En revanche, à la différence de l'emploi temporel prototypique, ce n'est pas

Revue de Sémantique et Pragmatique, 38 | 2015 
le procès au futur qui est placé dans l'ultériorité de to (à savoir le fait que l'objet se trouve à un endroit donné), d'où le fait que nous qualifions cet emploi d'énonciativotemporel, au même titre que l'emploi d'énonciation analysé dans la section précédente. Le locuteur se met à la place de l'interlocuteur et situe le procès au moment où celui-ci découvrira l'objet à l'endroit indiqué. Le fait que le locuteur accompagne l'interlocuteur au moins sur une partie du déplacement comme l'indique L. de Saussure pour (3) ou comme nous avons pu le constater pour (4) renforce notre hypothèse selon laquelle le locuteur adopte le point de vue ultérieur de son interlocuteur. Deux éléments doivent encore être expliqués : pourquoi cet emploi est toujours réalisé avec être et avoir et pourquoi il ne l'est jamais au futur antérieur. Le premier élément s'explique en deux temps. Dans un premier temps, si on ne trouve d'énoncés à sens de découverte qu'avec être et avoir, c'est parce que cet emploi nécessite d'avoir des verbes d' état, c'est-à-dire qui expriment des procès sans bornes, et peuvent donc être vrais notamment au moment de l'énonciation principale to. Or, on rappelle que l'emploi de découverte consiste pour le locuteur-énonciateur à indiquer à l'interlocuteur l'endroit où se trouve l'objet que ce dernier recherche. De ce fait, l'effet de sens de découverte est produit avec un procès qui est considéré comme vrai au moment de l'énonciation principale par le locuteur-énonciateur, à savoir le fait que l'objet se trouve bel et bien à cet endroit-là au moment où il parle. Dans un deuxième temps, si parmi les verbes d'états, seuls être et avoir semblent réaliser cet effet de sens, c'est parce qu'en français, l'expression de la localisation d'un être (ou un objet) dans un lieu donné se fait essentiellement avec être (« les oeufs sont au rayon frais ») ou avoir (« il y a des oeufs au rayon frais »). La restriction qui porte sur les verbes avec lesquels on rencontre cet emploi est donc inhérente à sa définition même. Le deuxième élément à expliquer est le fait que l'emploi de découverte semble exclure le futur antérieur. Comme pour la restriction concernant le type de verbe, celle-ci est également imputable à la définition même de cet emploi. En effet, comme on l'a déjà évoqué, dans ce type d'énoncé le locuteur-énonciateur évoque le moment où l'interlocuteur entrera en contact visuel avec l'objet recherché. Or, je ne peux voir qu'un objet qui est là, non un objet qui $a$ été là. De ce fait, le temps verbal qui dénote le procès exprimant la localisation de l'objet doit nécessairement le montrer en tension (au sens de Guillaume 1929/1970), c'est-àdire en accomplissement. C'est ce que permet non seulement le futur simple, mais aussi la forme périphrastique. La forme périphrastique, comme on l'a mis en évidence dans la section 1 , situe la phase préparatoire du procès dans le PRÉSENT de l'énonciation principale. Par inférence, l'accomplissement du procès lui-même est situé dans l'ultériorité du PRÉSENT, à savoir le FUTUR. En revanche, le futur antérieur, parce qu'il saisit le procès en extension, c'est-à-dire après son accomplissement, ne peut entrer en jeu dans la production d'un effet de sens qui implique que le procès soit saisi en accomplissement.

\section{Conclusion}

Nous avons réuni dans la même étude les deux emplois d'énonciation et de découverte au motif de ce que dans les deux cas le futur porte non sur le procès de l'énoncé mais sur un acte d'énonciation ultérieur pour l'emploi d'énonciation; sur un acte de perception ultérieur pour l'emploi de découverte. Dans les deux cas, le futur entre en jeu dans un effet de sens qui permet de situer un élément dans l'ultériorité (un acte d'énonciation ou un acte de perception) et non d'exprimer l'attitude du locuteur par rapport au 
contenu propositionnel de l'énoncé en montrant le procès comme simplement possible ou probable. En ce sens, ces deux emplois ne peuvent être considérés comme des emplois modaux épistémiques et sont définis comme des emplois énonciativotemporels.

\section{BIBLIOGRAPHIE}

Abouda, L. (2004), « De la polyphonie à la 'polychronie'. Un modèle de traitement de la polysémie temporelle. Application au futur ", in Suso, J. et López, R. (coord.), Le français face aux défis actuels. Histoire, langue et culture, 149-160.

Azzopardi, S. (sous presse), « Le futur est-il un marqueur modal ? Analyse du fonctionnement du futur à effet de sens « conjectural» en français et en espagnol », inBaranzini L., Sanchez Mendez J. \& Saussure L. de (éds), Le futur dans les langues romanes, Bruxelles, Bern, Berlin, Frankfurt am Main, New York, Oxford, Wien : Peter Lang.

Azzopardi, S. (2011), Le Futur et le Conditionnel : valeur en langue et effets de sens en discours. Analyse contrastive espagnol / français, Montpellier : Université de Montpellier 3 : thèse de doctorat, Sciences du langage, sous la direction de Bres, Jacques. [en ligne sur Internet]. Disponible sur http://www.biu-montpellier.fr/florabium/jsp/nnt.jsp?nnt=2011MON30052.

Azzopardi, S. et Bres, J. (2011), « Temps verbal et énonciation. Le futur et le conditionnel en français : l'un est dialogique, l'autre pas (souvent) », Cahiers de praxématique, 56, 55-78.

Bakhtine, M. (1952/1979/1984), « Les genres du discours », in Esthétique de la création verbale, Paris : Gallimard, 265- 308.

Barbéris, J-M., Bres, J. et Siblot, P. (1998), De l'actualisation, Paris : CNRS- Editions.

Bres, J. (2009), « Dialogisme et temps verbaux de l'indicatif », Langue Française, 163, 21-39.

Bres, J. (2003), « Temps verbal, aspect et point de vue : de la langue au discours », Cahiers de praxématique, $41,55-84$.

Bres, J. et Azzopardi, S. (2012), « On aurait oublié les clés du dialogisme sur la porte de l'analyse ? De l'effet de sens de conjecture du futur et du conditionnel en français ", in Bres, J., Nowakowska, A., Sarale, J.-M. et Sarrazin, S. (eds) (2012), 135-147.

Bres, J. et Nowakowska, A. (2006), « Dialogisme : du principe à la matérialité discursive », in Perrin, L. (ed.) (2006), 21-48.

Bybee, J., Perkins, R. et Pagliuca, W. (1994), The evolution of grammar, Chicago : The University of Chicago Press.

Damourette, J. et Pichon, E. (1911-1936), Des mots à la pensée : essai de grammaire de la langue française, Paris : d'Artrey.

Dendale, P. (2001), « Le futur conjectural versus devoir épistémique : différences de valeur et restrictions d'emploi », Le Français Moderne, 69, 1, 1-20.

Gosselin, L. (2005), Temporalité et modalité, Bruxelles : De Boeck Duculot. 
Gosselin, L. (1996), Sémantique de la temporalité en français : un modèle calculatoire et cognitif du temps et de l'aspect, Louvain-la-Neuve : Duculot.

Guillaume, G. (1929/1970), Temps et verbe, Paris : Champion.

Hopper, P. et Traugott, E. (1993/2003), Grammaticalization, Cambridge: Cambridge University Press.

Kronning, H. (2003), « Modalité et évidentialité », in Birkelund, M., Boysen, G. \&

Kjærsgaard, P. S. (eds) (2003), Aspects de la Modalité, Tübingen : Max Niemeyer, Linguistische Arbeiten 469, 131-151.

Kronning, H. (1993), « Modalité et temps : devoir + infinitif périphrase du futur », in Actes du XIIe Congrès des Romanistes Scandinaves, Aalborg, 11-15 août 1993, (éd. G. Boysen), Vol. I, Aalborg 1994, 283-295.

Martin, R. (1985), « Langage et temps de dicto », Langue Française, 67, 23-37.

Martin, R. (1981), « Le futur linguistique : temps linéaire ou temps ramifié ? », Langages, 64, 81-91.

Pustejovsky, J. (1995), The Generative Lexicon, Cambridge (MA): The MIT Press.

Saussure, L. de (2012), « Modalité épistémique, évidentialité et dépendance contextuelle », Langue française, 173, 131-143.

Saussure, L. de et Morency, P. (2012), « A cognitive pragmatic view of the French epistemic Future », Journal of French Language Studies, 22, 207-223.

Sperber, D. et Wilson, D. (2nd edition) (1995), Relevance: Communication and Cognition, Oxford: Blackwell.

Swart, H. de (1998), « Aspect Shift and Coercion », Natural Language and Linguistic Theory, 16: 347-385.

Vuillaume, M. (1998), « Le discours indirect libre et le passé simple », in. Vogeleer, S. (ed) (1998), Temps et discours, BCILL 99, Louvain-la-Neuve : Peeters, 191-201.

\section{NOTES}

1. Nous utilisons les majuscules pour désigner les époques et les minuscules pour faire référence aux temps verbaux.

2. Dont l'emploi de conjecture, sans doute, a été le plus étudié (i.a. Damourette et Pichon, 1911-1936 ; Martin, 1981 ; Dendale, 2001 ; Bres et Azzopardi, 2012).

3. Pour une définition plus développée de cette notion, on renvoie à Barbéris, Bres et Siblot (1998).

4. Nous employons le terme co(n)texte pour désigner à la fois le cotexte et le contexte.

5. Pour un type d'approche en partie similaire de certains emplois non temporels du futur dans les cadres de la polyphonie, cf Kronning (1993 et 2003, i.a.) et Abouda (2004) qui introduit le concept de "polychronie».

6. Voir également le modèle calculatoire de Gosselin $(1996,2005)$ qui pose lui aussi un ensemble d'instructions temporelles et aspectuelles basé sur la morphologie des temps verbaux.

7. Ou, mais plus difficilement, par du discours direct : (1b) : c'est un oncle qui me demande, dirat-elle 
8. La prise en charge de l'explication par le locuteur est inhérente à la nature abductive du raisonnement qui conduit à formuler l'hypothèse la plus plausible au sujet d'une situation (Sur ce point cf. Dendale 2001, Azzopardi 2011 et Azzopardi sous presse).

9. Eclaircissons les enchâssements énonciatifs : Juliana raconte à son fils Pierre (interaction 1) qu'Odette, fille de Gaston, lui a dit (interaction 2) qu'elle était très mécontente que son père raccompagne la belle Lucette à Alès parce qu'elle imaginait qu'ensuite il lui dirait (interaction 3) qu'il était fatigué, qu'il avait mal à la tête, etc... comme prétexte pour ne pas aller à la mer.

10. Notons que cependant le passé composé peut également être porteur, suivant le co(n)texte, d'un acte d'énonciation implicite ultérieur : c'est le cas dans le premier tour de parole de (10) : (10) Elle. N'use pas ta salive, je sais ce que tu vas me dire. (très simple). Je me suis fait peloter. ( $\rightarrow$ tu vas me dire que je me suis fait peloter).

11. Mais pas l'exclusive qu'elle pose : le passé composé (note 5) est également apte à un emploi en discours indirect libre.

\section{RÉSUMÉS}

Quand le futur ne porte pas sur le procès qu'il actualise : futur d'énonciation et futur de découverte Dans ce travail, qui s'inscrit dans les cadres d'une linguistique de l'actualisation et fait appel à la notion énonciative de dialogisme, nous réunissons deux emplois du futur (simple, antérieur et périphrastique) en français dont le fonctionnement est distinct de l'emploi prototypique temporel sans pour autant être un emploi exprimant une modalité. Il s'agit des emplois d'énonciation et de découverte, emplois énonciativo-temporels, dans lesquels le futur ne porte pas sur le procès qu'il actualise mais sur une énonciation secondaire (emplo d'énonciation) ou sur un point de vue distinct de celui du locuteur-énonciateur principal (emploi de découverte).

Two specific usages of the future tenses in French : enunciative future and discovery future. This paper investigates two usages of the future tenses (simple, anterior and periphrastic ones) in French : the enunciative future and the discovery future. Both are different from the prototypical temporal usage of French future tenses but are not modal ones. We call them temporalenunciative usages. Using the concept of process of actualization and the enunciative notion of dialogism, it is found that in these two usages the future tense isn't used to locate the event but to locate a secondary enunciation (enunciative usage) or a different point of view from the principal enunciator's one (discovery usage).

\section{INDEX}

Mots-clés : futur, dialogisme, énonciation, emplois spécifiques

Keywords : future tenses, dialogism, enunciation, specific usages 


\section{AUTEURS}

SOPHIE AZZOPARDI

CLILLAC-ARP EA3967, Université Paris Diderot Paris 7

JACQUES BRES

Praxiling UMR5267 CNRS, Université Paul Valéry Montpellier 3 